\title{
Generalized Interlacing InEQUALities
}

\author{
Chi-Kwong Li* Yiu-Tung Poon $†$ and Nung-Sing SzE ${ }^{\ddagger}$
}

\section{In memory of Professor Ky Fan}

\begin{abstract}
We discuss some applications of generalized interlacing inequalities of Ky Fan to the study of (a) some classical matrix inequalities and (b) matrix problems in quantum information science.
\end{abstract}

AMS Classification 15A18, 15A57, 15A60, 15A90.

Keywords Hermitian matrices, eigenvalues, interlacing inequalities, numerical ranges.

\section{Introduction}

Professor Ky Fan was a great scholar and educator. Not only did he obtain many interesting and useful results, he was also eager to help young colleagues and shared his insights and experience about mathematical research and education. The first author met him in several occasions and received valuable advice from him. For instance, he emphasized that "One should keep an open mind about research." "Interesting mathematical results often build on simple assumptions but have deep implications." Professor Fan had many results of this nature. For example, he and Pall [7] obtained the following generalized interlacing inequalities for eigenvalues of complex Hermitian (real symmetric) matrices that have many applications as shown in later sections.

Theorem 1.1 Let $a_{1} \geq \cdots \geq a_{n}$ and $b_{1} \geq \cdots \geq b_{k}$ be real numbers and $1 \leq k<n$. There exists an $n \times n$ Hermitian matrix $A$ with a $k \times k$ principal submatrix $B$ having eigenvalues $a_{1} \geq \cdots \geq a_{n}$ and $b_{1} \geq \cdots \geq b_{k}$, respectively, if and only if

$$
a_{j} \geq b_{j} \geq a_{n-k+j}, \quad j=1, \ldots, k .
$$

Here, we present a proof for completeness. Denote by $M_{n}$ the set of $n \times n$ complex matrices and $H_{n}$ the set of Hermitian matrices in $M_{n}$.

\footnotetext{
*Department of Mathematics, The College of William and Mary, Williamsburg, Virginia 23187, USA (ckli@math.wm.edu). This research was done when Li was visiting the Department of Mathematics, Hong Kong University of Science and Technology under a Fulbright Fellowship in 2011. Li is an honorary professor of the University of Hong Kong, Taiyuan University of Technology, Shanghai University. His research was supported by an NSF grant, a HK RGC grant, and the Shanxi 100 talent program.

${ }^{\dagger}$ Department of Mathematics, Iowa State University, Ames, IA 50011 (ytpoon@iastate.edu). Research of Poon was supported by an NSF grant.

${ }^{\ddagger}$ Department of Applied Mathematics, The Hong Kong Polytechnic University, Hung Hom, Hong Kong (raymond.sze@inet.polyu.edu.hk). Research of Sze was supported by a HK RGC grant.
} 
Proof of Theorem 1.1. First consider the case when $k=n-1$, and $B$ is obtained from $A$ by deleting its first row and first column. We need only address the case $b_{j} \in\left(a_{j+1}, a_{j}\right)$ for $j=1, \ldots, n-1$, since the general case $b_{j} \in\left[a_{j+1}, a_{j}\right]$ follows by a continuity argument.

Let $U$ be unitary such that $A=U^{*} \operatorname{diag}\left(a_{1}, \ldots, a_{n}\right) U$. If $u=\left(u_{1}, \ldots, u_{n}\right)^{t}$ is the first column of $U$, then the $(1,1)$ entry of $(t I-A)^{-1}=U^{*} \operatorname{diag}\left(t-a_{1}, \ldots, t-a_{n}\right)^{-1} U$ equals

$$
f(t)=\sum_{j=1}^{n}\left|u_{j}\right|^{2}\left(t-a_{j}\right)^{-1}
$$

which is continuous in each interval $\left(a_{j+1}, a_{j}\right)$ for $j=1, \ldots, n-1$, and satisfies $\lim _{t \rightarrow a_{j+1}^{+}} f(t)=\infty$ and $\lim _{t \rightarrow a_{j}^{-}} f(t)=-\infty$. Thus, $f$ has at least one zero in $\left(a_{j+1}, a_{j}\right)$ for $j=1, \ldots, n-1$. Now, by the adjoint formula for the inverse, it is the same as

$$
f(t)=\operatorname{det}(t I-B) / \operatorname{det}(t I-A)=\left(\prod_{j=1}^{n-1}\left(t-b_{j}\right)\right) /\left(\prod_{j=1}^{n}\left(t-a_{j}\right)\right) .
$$

We conclude that $b_{j}$ is the unique zero in $\left(a_{j+1}, a_{j}\right)$ for $j=1, \ldots, n-1$.

If $k<n-1$, we can repeat the above argument $n-k$ times on a sequence of submatrices of $A$ by removing one row and one column each time to get the desired inequalities.

To prove the converse, we first consider the case $k=n-1$. We use the proof by Mirsky [19] described in Marshall and Olkin [18].

We aim at constructing $A=\left[\begin{array}{cc}D & v \\ v^{t} & v_{n}\end{array}\right]$, where $D=\operatorname{diag}\left(b_{1}, \ldots, b_{n-1}\right)$ is a diagonal matrix and $v=\left(v_{1}, \ldots, v_{n-1}\right)^{t}$ is a real vector. Then for $f(t)=\operatorname{det}(t I-A)=\prod_{j=1}^{n}\left(t-a_{j}\right)$ and $g(t)=$ $\operatorname{det}(t I-D)=\prod_{j=1}^{n-1}\left(t-b_{j}\right)$ we have

$$
f(t)=g(t)\left(t-v_{n}-v^{t}(t I-D)^{-1} v\right)=g(t)\left(t-v_{n}-\sum_{j=1}^{n-1} \frac{v_{j}^{2}}{t-b_{j}}\right) .
$$

Let $v_{n}=\sum_{j=1}^{n} a_{j}-\sum_{j=1}^{n-1} b_{j}$, and for $j=1, \ldots, n-1, v_{j} \geq 0$ such that

$$
v_{j}^{2}=-f\left(b_{j}\right) / g^{\prime}\left(b_{j}\right)=-\frac{\prod_{r=1}^{n}\left(b_{j}-a_{r}\right)}{\prod_{r \neq j}\left(b_{j}-b_{r}\right)},
$$

which is positive because $b_{r}-a_{n}>0, b_{r}-a_{r}<0$, and $\left(b_{j}-a_{r}\right) /\left(b_{j}-b_{r}\right)>0$ for all $j \neq r$, by our assumption. Then the polynomial $f(t)-g(t)\left(t-v_{n}-\sum_{j=1}^{n-1} v_{j}^{2} /\left(t-b_{j}\right)\right)$ must be the zero polynomial because it has degree $n-2$, and has zeros at $b_{1}, \ldots, b_{n-1}$. Thus, we get the desired matrix $A$.

Now, suppose $k<n-1$. We can use induction on $n-k$. One can find $\tilde{a}_{1} \geq \cdots \geq \tilde{a}_{n-1}$ such that

$$
\min \left\{a_{j}, b_{k-n+j+1}\right\} \geq \tilde{a}_{j} \geq \max \left\{a_{j+1}, b_{j}\right\}, \quad j=1, \ldots, n-1,
$$

here $b_{k-n+j+1}=a_{j}$ if $k-n+j+1 \leq 0$. For instance, one can define

$$
\tilde{a}_{j}= \begin{cases}\max \left\{a_{j+1}, b_{j}\right\} & 1 \leq j \leq k \\ \min \left\{a_{j}, b_{k-n+j+1}\right\} & k<j<n\end{cases}
$$


Then

$$
a_{1} \geq \tilde{a}_{1} \geq a_{2} \geq \cdots \geq a_{n-1} \geq \tilde{a}_{n-1} \geq a_{n}
$$

and

$$
\tilde{a}_{j} \geq b_{j} \geq \tilde{a}_{n-k-1+j}, \quad j=1, \ldots, k .
$$

By the induction assumption, we can construct a matrix $\tilde{A} \in H_{n-1}$ with eigenvalues $\tilde{a}_{1} \geq \cdots \geq$ $\tilde{a}_{n-1}$, whose $k \times k$ leading principal submatrix has eigenvalues $b_{1} \geq \cdots \geq b_{k}$, and $\tilde{A}$ is a principal submatrix of $A \in H_{n}$ such that $A$ has eigenvalues $a_{1} \geq \cdots \geq a_{n}$.

\section{Some applications to matrix inequalities}

The generalized interlacing inequalities have many nice applications to matrix inequalities.

Theorem 2.1 Suppose $A$ is an $n \times n$ Hermitian matrix with eigenvalues $a_{1} \geq \cdots \geq a_{n}$ and diagonal entries $d_{1} \geq \cdots \geq d_{n}$. Then $\left(d_{1}, \ldots, d_{n}\right) \prec\left(a_{1}, \ldots, a_{n}\right)$, i.e., $\sum_{j=1}^{k} d_{j} \leq \sum_{j=1}^{k} a_{j}$ for $k=1, \ldots, n$, and the equality holds when $k=n$.

Proof. Consider the $k \times k$ submatrix $B$ of $A$ with diagonal entries $d_{1}, \ldots, d_{k}$. Suppose $B$ has eigenvalues $b_{1} \geq \cdots \geq b_{k}$. Then $\sum_{j=1}^{k} d_{j}=\sum_{j=1}^{k} b_{j} \leq \sum_{j=1}^{k} a_{j}$ by the interlacing inequalities.

Denote by $\lambda_{1}(A) \geq \cdots \geq \lambda_{n}(A)$ the eigenvalues of an $n \times n$ Hermitian matrix $A$. Suppose $E$ is a small perturbation of $A$ due to numerical errors and measuring errors, etc. We can compare the eigenvalues of $A+E$ and those of $A$. By the result of Liskii, Mirsky, and Wielandt, see e.g. [1, Theorem 9.4] or [22, Theorem IV.4.8], we have

$$
\sum_{j=1}^{k} \lambda_{n-j+k}(E) \leq \sum_{j=1}^{k}\left(\lambda_{i_{j}}(A+E)-\lambda_{i_{j}}(A)\right) \leq \sum_{j=1}^{k} \lambda_{j}(E) \quad \text { for any } 1 \leq i_{1}<\cdots<i_{k} \leq n .
$$

One can see [13] for a simple proof, and [12] for results on perturbation on structured Hermitian matrices, where the generalized interlacing inequalities also play key roles in the proof. Here, we present the results and proofs of the Thompson's inequalities, which are important special cases for the complete set of inequalities by Horn, Klyascho, Fulton, etc., see e.g., [8].

Theorem 2.2 Suppose $A, B$ and $C=A+B$ are Hermitian matrices. Then for any $1 \leq i_{1}<\cdots<$ $i_{k} \leq n$ and $1 \leq j_{1}<\cdots<j_{k} \leq n$ with $i_{k}+j_{k}-k \leq n$, we have

$$
\sum_{r=1}^{k} \lambda_{i_{r}}(A)+\sum_{r=1}^{k} \lambda_{j_{r}}(B) \geq \sum_{r=1}^{k} \lambda_{i_{r}+j_{r}-r}(C)
$$

Proof. We prove by induction on $n$. Clearly, the result holds when $n=1$. Assume $n>1$. First the result is clear if $k=n$. Let $p$ be the largest integer such that $i_{t}=t$ for $t=1, \ldots, p$, and let $q$ be the largest integer such that $j_{t}=t$ for $t=1, \ldots, q$. Notice that $p \leq k$ and $q \leq k$. Exchanging $A$ and $B$ if necessary, we may assume that $q \leq p$. We can further assume that $i_{k}<n$. Suppose not. 
Then $i_{k}=n$ and $i_{k}+j_{k}-k \leq n$ imply that $j_{k}=k$ and hence $p=q=k$. So, one can exchange $A$ and $B$ again so that $i_{k}=k<n$.

For simplicity, let $a_{j}=\lambda_{j}(A), b_{j}=\lambda_{j}(B)$, and $c_{j}=\lambda_{j}(C)$ for $j=1, \ldots, n$. Let $\left\{u_{1}, \ldots, u_{n}\right\}$ and $\left\{v_{1}, \ldots, v_{n}\right\}$ be orthonormal sets of eigenvectors of $B$ and $C$ such that

$$
B u_{j}=b_{j} u_{j} \quad \text { and } \quad C v_{j}=c_{j} v_{j}, \quad j=1, \ldots, n .
$$

Let $\mathcal{S}$ be an $(n-1)$-dimensional subspace containing the vectors $v_{1}, \ldots, v_{q}, u_{q+2}, \ldots, u_{n}$ and $P$ be the orthogonal projection of $\mathbb{C}^{n}$ onto $\mathcal{S}$. Identify $\tilde{A}=P A P, \tilde{B}=P B P$ and $\tilde{C}=P C P$ as matrices in $H_{n-1}$. Then their eigenvalues $\tilde{a}_{1} \geq \cdots \geq \tilde{a}_{n-1}, \tilde{b}_{1} \geq \cdots \geq \tilde{b}_{n-1}, \tilde{c}_{1} \geq \cdots \geq \tilde{c}_{n-1}$, interlace, respectively, those of $A, B$ and $C$. By the induction assumption on $\tilde{C}=\tilde{A}+\tilde{B}$ with the sequences $1 \leq i_{1}<\cdots<i_{k} \leq n-1$ and $1 \leq j_{1}<\cdots<j_{q}<j_{q+1}-1<\cdots<j_{k}-1 \leq n-1$, we have

$$
\sum_{s=1}^{q} \tilde{c}_{i_{s}+j_{s}-s}+\sum_{s=q+1}^{k} \tilde{c}_{i_{s}+j_{s}-s-1} \leq \sum_{s=1}^{k} \tilde{a}_{i_{s}}+\sum_{s=1}^{q} \tilde{b}_{j_{s}}+\sum_{j=q+1}^{k} \tilde{b}_{j_{s}-1} .
$$

By the interlacing inequality, we see that $\tilde{a}_{i_{s}} \leq a_{i_{s}}$ for $s=1, \ldots, k ; \tilde{b}_{j_{s}} \leq b_{j_{s}}$ for $s=1, \ldots, q$; $\tilde{c}_{i_{s}+j_{s}-s-1} \geq c_{i_{s}+j_{s}-s}$ for $s=q+1, \ldots, k$. Since $\mathcal{S}$ contains $v_{1}, \ldots, v_{q}$, it follows that $c_{1}, \ldots, c_{q}$ are eigenvalues of $\tilde{C}$, and by interlacing $c_{t}=\tilde{c}_{t}$ for $t=1, \ldots, q$. Moreover, for $s=1, \ldots, q$, we have $i_{s}=j_{s}=s$, and thus, $i_{s}+j_{s}-s=s$. It follows that

$$
\sum_{s=1}^{q} \tilde{c}_{i_{s}+j_{s}-s}=\sum_{s=1}^{q} c_{i_{s}+j_{s}-s}
$$

Since $\mathcal{S}$ contains $u_{q+2}, \ldots, u_{n}$, it follows that $b_{q+2}, \ldots, b_{n}$ are eigenvalues of $\tilde{B}$, and by interlacing $b_{t}=\tilde{b}_{t-1}$ for $t=q+2, \ldots, n$. As $j_{q+1}>q+1, b_{j_{s}}=\tilde{b}_{j_{s}-1}$ for $s \geq q+1$. Thus,

$$
\sum_{s=q+1}^{k} \tilde{b}_{j_{s}-1}=\sum_{s=q+1}^{k} b_{j_{s}}
$$

Combining these facts with (2), we get the desired inequalities.

\section{Applications in quantum information science}

In this section, we describe some applications of the generalized interlacing inequalities to problems in quantum information science. Here, we briefly describe the background and refer the readers to [4] for details.

Quantum states are represented as density matrices in $M_{n}$, i.e., positive semidefinite matrices with trace one. A quantum channel is represented as a completely positive linear map on $M_{n}$ with an operator sum representation

$$
L(X)=\sum_{j=1}^{r} F_{j} X F_{j}^{*}
$$


for some matrices $F_{1}, \ldots, F_{r}$ known as the error operators. A map $L$ is said to be a completely positive map if the map $I_{k} \otimes L$ is a positive map for all $k$, see [3]. One would like to find a recovery channel $T$ such that $T \circ L(X)=X$ whenever $P X P=X$ for some orthogonal projection $P$. The range space of $P$ is known as a quantum error correction code of the channel $L$. One would like to find $P$ with a maximum rank for the given channel $L$. For a given channel $L$ with the operator sum representation described in (3), it is known that the range space of an orthogonal projection $P$ is a quantum error correction code corresponding to $L$ if and only if there are scalars $\gamma_{i j} \in \mathbb{C}$ such that

$$
P F_{i}^{*} F_{j} P=\gamma_{i j} P \quad \text { for all } 1 \leq i, j \leq r,
$$

see, e.g., [20, Chapter 10]. This leads to the study of the rank- $k$ numerical range of $A \in M_{n}$ defined by

$$
\Lambda_{k}(A)=\left\{\gamma \in \mathbb{C}: U^{*} A U=\left[\begin{array}{cc}
\gamma I_{k} & * \\
* & *
\end{array}\right] \text { for some unitary } U\right\}
$$

When $k=1$, the definition reduces to the classical numerical range

$$
W(A)=\left\{x^{*} A x: x \in \mathbb{C}^{n}, x^{*} x=1\right\}
$$

of $A \in M_{n}$, which is very useful in the study of matrices and operators; see [9, Chapter 1$]$.

\subsection{Higher rank numerical ranges}

In [15], the following theorem was proved which confirmed some conjectures in [4].

Theorem 3.1 Let $A \in M_{n}$. Then

$$
\Lambda_{k}(A)=\left\{\gamma \in \mathbb{C}: e^{i \xi} \gamma+e^{-i \xi} \bar{\gamma} \leq \lambda_{k}\left(e^{i \xi} A+e^{-i \xi} A^{*}\right) \text { for all } \xi \in[0,2 \pi)\right\} .
$$

Consequently, $\Lambda_{k}(A)$ is always convex. If $A$ is normal with eigenvalues $a_{1}, \ldots, a_{n}$, then

$$
\Lambda_{k}(A)=\bigcap_{1 \leq j_{1}<\cdots<j_{n-k+1} \leq n} \operatorname{conv}\left\{a_{j_{1}}, \ldots, a_{j_{n-k+1}}\right\}
$$

A key step of the proofs of (4) is the use of the generalized interlacing inequalities. To be precise, in the proof one has to show that zero is in the rank- $k$ numerical range of a matrix $B$ with eigenvalues $1+i \mu_{1}, \ldots, 1+i \mu_{k}$ and $-1+i \mu_{k+1}, \ldots,-1+i \mu_{n}$, where $\mu_{1}, \ldots, \mu_{n}$ are real numbers satisfying

$$
-\mu_{n}>\mu_{1}, \quad-\mu_{n-1}>\mu_{2}, \quad \ldots, \quad-\mu_{n-k+2}>\mu_{k-1}, \quad-\mu_{n-k+1}>\mu_{k}
$$

and

$$
-\mu_{k+1} \leq \mu_{k}, \quad-\mu_{k+2} \leq \mu_{k-1}, \quad \ldots, \quad-\mu_{2 k-1} \leq \mu_{2}, \quad-\mu_{2 k} \leq \mu_{1} .
$$

To complete the proof, one has to apply Theorem 1.1 and conclude that there is a unitary $U$ such that $U^{*} B U$ has a principal submatrix $0_{k}$, and hence $0 \in \Lambda_{k}(B)$.

The following result gives the optimal bound on $n$ for $\Lambda_{k}(A)$ to be non-empty. 
Theorem 3.2 The set $\Lambda_{k}(A)$ is non-empty if one of the following conditions is satisfied.

$$
\text { (I) } A \in H_{n} \text {, with } n \geq 2 k-1 \text {. (II) } A \in M_{n} \text {, with } n \geq 3 k-2 \text {. }
$$

Furthermore, the bounds in (I) and (II) are optimal.

Proof. The condition (I) was proved in [6]. Here we give a proof using Theorem 1.1. Suppose $A \in H_{n}$, with $n \geq 2 k-1$. We may assume that $A$ has eigenvalues $a_{1} \geq a_{2} \geq \cdots \geq a_{n}$. By Theorem 1.1, there is a unitary $U$ such that $U^{*} A U$ has a leading principal submatrix equal to $a_{k} I_{k}$.

Suppose $n<2 k-1$. Let $A=\operatorname{diag}(1,2, \ldots, n)$. If $U^{*} A U$ has a leading principal submatrix equal to $b I_{k}$, then $a_{j} \geq b \geq a_{n-j+1}$ for $j=1, \ldots, k$, which is impossible. Thus, $\Lambda_{k}(A)$ is empty.

The condition (II) was proved in [14, Theorems 1 and 3].

\section{$3.2(p, k)$ numerical range}

In [5], for a better error correction scheme, the authors consider a special decomposition of the matrix space. Given a quantum channel $L$ with the operator sum representation in (3), they would like to find a recovery channel $R$ such that for each $B \in M_{k}$,

$$
R \circ L\left(\left(I_{p} \otimes B\right) \oplus 0_{n-p k}\right)=\left(A_{B} \otimes B\right) \oplus 0_{n-p k} \quad \text { for some } A_{B} \in M_{p} .
$$

Fixed an arbitrary orthonormal basis $\left\{e_{1}, \ldots, e_{p}\right\}$ in $\mathbb{C}^{p}$. The authors showed that such recovery channel $R$ exists if and only if there are scalars $\gamma_{i j r s} \in \mathbb{C}$ such that

$$
P_{k k} F_{i}^{*} F_{j} P_{\ell \ell}=\gamma_{i j r s} P_{k \ell} \quad \text { for all } 1 \leq i, j \leq r \text { and } 1 \leq k, \ell \leq p,
$$

where $P_{k \ell}=\left(e_{k} e_{\ell}^{*} \otimes I_{k}\right) \oplus 0_{n-p k}$; see [11] and also [5]. This leads to an extension of rank-k numerical range to the $(p, k)$ numerical range of $A \in M_{n}$ defined by

$$
\Lambda_{p, k}(A)=\left\{B \in M_{p}: U^{*} A U=\left[\begin{array}{cc}
B \otimes I_{k} & * \\
* & *
\end{array}\right] \text { for some unitary } U\right\} .
$$

It is easy to see that

$$
\Lambda_{p, k}(A)=\left\{B \in M_{p}: \text { there is } X \text { such that } X^{*} X=I_{p k} \text { and } X^{*} A X=B \otimes I_{k}\right\} .
$$

When $p=1$, we get the rank- $k$ numerical range; when $k=1$, we get the $p$ th matricial range (see [16]); when $p=k=1$, we get the classical numerical range (see [9]).

The following theorem is easy to verify.

Theorem 3.3 Let $A \in M_{n}$.

(a) $\Lambda_{p, k}\left(\alpha A+\beta I_{n}\right)=\alpha \Lambda_{p, k}(A)+\beta I_{p}$ for any $\alpha, \beta \in \mathbb{C}$.

(b) $\Lambda_{p, k}\left(X^{*} A X\right) \subseteq \Lambda_{p, k}(A)$ for any $n \times m$ matrix $X$ with $X^{*} X=I_{m}$, and the equality holds if $m=n$.

(c) A matrix $B \in M_{p}$ satisfies $B \in \Lambda_{p, k}(A)$ if and only if $V^{*} B V \in \Lambda_{p, k}(A)$ for any unitary $V \in M_{p}$. So, $\Lambda_{p, k}(A)$ is a union of unitary similarity orbits $\left\{V^{*} B V: V\right.$ is unitary $\}$ in $M_{p}$. 
The following theorem gives a necessary condition for $B \in \Lambda_{p, k}(A)$.

Theorem 3.4 Let $A \in M_{n}$. If $B \in \Lambda_{p, k}(A)$, then

$$
\Lambda_{n-p k+1}(A) \subseteq \Lambda_{1}(B) \subseteq \Lambda_{k}(A)
$$

Proof. Suppose there is a unitary $U$ such that $U^{*} A U$ has a principal submatrix of the form $\tilde{B}=B \otimes I_{k}$ with $B \in M_{p}$. Then for each $\xi \in[0,2 \pi)$, we can apply Theorem 1.1 to conclude that

$$
\begin{aligned}
\lambda_{k}\left(e^{i \xi} A+e^{-i \xi} A^{*}\right) & \geq \lambda_{k}\left(e^{i \xi} \tilde{B}+e^{-i \xi} \tilde{B}^{*}\right)=\lambda_{1}\left(e^{i \xi} B+e^{-i \xi} B^{*}\right) \\
& =\lambda_{1}\left(e^{i \xi} \tilde{B}+e^{-i \xi} \tilde{B}^{*}\right) \geq \lambda_{n-k p+1}\left(e^{i \xi} A+e^{-i \xi} A^{*}\right) .
\end{aligned}
$$

Consequently, if we obtain convex sets $\Lambda_{n-k p+1}(A), \Lambda_{1}(B)$, and $\Lambda_{k}(A)$ using the half spaces described in Theorem 3.1, then the half space containing $\Lambda_{n-k p+1}(A)$ will be a subset of the half space containing $\Lambda_{1}(B)$, which in turns will be a subset of the half space containing $\Lambda_{k}(A)$. Taking the intersection, we get the asserted inclusion relations.

For a general matrix $A \in M_{n}$, it is difficult to check whether a given matrix $B \in M_{p}$ belongs to $\Lambda_{p, k}(A)$. If $A$ is Hermitian, we have a necessary and sufficient condition for $B \in \Lambda_{p, k}(A)$ in terms of the eigenvalues of $A$ and those of $B$.

Theorem 3.5 Let $A \in H_{n}$ and $p k \leq n$. Then $B \in \Lambda_{p, k}(A)$ if and only if the eigenvalues of $B \otimes I_{k}$ and $A$ satisfy the generalized interlacing inequalities. In other words, $B \in \Lambda_{p, k}(A)$ if and only if

$$
\lambda_{n-(p-j+1) k+1}(A) \leq \lambda_{j}(B) \leq \lambda_{j k}(A) \quad \text { for all } j=1, \ldots, p .
$$

Consequently, $\Lambda_{p, k}(A) \neq \emptyset$ if and only if

$$
\lambda_{j k}(A) \geq \lambda_{n-(p-j+1) k+1}(A) \quad \text { for all } j=1, \ldots, p .
$$

If $n \geq(p+1) k-1$, then the above inequalities always hold and hence $\Lambda_{p, k}(A)$ is always non-empty. If $n<(p+1) k-1$, then the above inequalities hold if and only if all of the inequalities become equalities so that $\Lambda_{p, k}(A)$ consists of matrices in $H_{p}$ with eigenvalues $a_{k}, a_{2 k}, \ldots, a_{p k}$.

Proof. Notice that $B \in \Lambda_{p, k}(A)$ if and only if $U^{*} A U$ has a principal submatrix with eigenvalues

$$
\underbrace{\lambda_{1}(B), \ldots, \lambda_{1}(B)}_{k}, \underbrace{\lambda_{2}(B), \ldots, \lambda_{2}(B)}_{k}, \ldots, \underbrace{\lambda_{p}(B), \ldots, \lambda_{p}(B)}_{k} .
$$

By Theorem 1.1, the first assertion follows. The last two assertions can be verified readily.

The following result provides bounds on $n$ ensuring that $\Lambda_{p, k}(A)$ is non-empty for any $A \in M_{n}$.

Theorem 3.6 If $n \geq 2(p+1) k-3$, then $\Lambda_{p, k}(A)$ is non-empty for any $A \in M_{n}$. 
Proof. Suppose $n \geq 2(p+1) k-3=2((p+1) k-1)-1$. Let $A=A_{1}+i A_{2}$, with $A_{1}, A_{2} \in H_{n}$. Then by Theorem 3.2, there exists a unitary $U \in M_{n}$ such that $U^{*} A_{1} U=\left[\begin{array}{cc}a I_{(p+1) k-1} & * \\ * & *\end{array}\right]$ for some $a \in \mathbb{R}$. Let $U^{*} A_{2} U=\left[\begin{array}{ll}B & * \\ * & *\end{array}\right]$ with $B \in H_{(p+1) k-1}$. By Theorem $3.5, \Lambda_{p, k}(B) \neq \emptyset$. Therefore, $\Lambda_{p, k}(A) \neq \emptyset$.

One may improve the bound $n$ if $A \in M_{n}$ has some special structure.

Theorem 3.7 Suppose $A \in M_{n}$ is unitarily similar to $A_{1} \oplus \ldots \oplus A_{p}$ such that $\Lambda_{k}\left(A_{j}\right) \neq \emptyset$ for all $j$. Then $\Lambda_{p, k}(A)$ is nonempty. Consequently, if each $A_{j}$ has dimension at least $3 k-2$, then $\Lambda_{p, k}(A)$ is nonempty. In particular, if $A \in M_{n}$ is normal with $n \geq(3 k-2) p$, then $\Lambda_{p, k}(A)$ is non-empty.

Proof. The given assumption ensures that each $A_{j}$ is unitarily similar to $\left[\begin{array}{cc}b_{j} I_{k} & * \\ * & *\end{array}\right]$. Thus, $\operatorname{diag}\left(b_{1}, \ldots, b_{k}\right) \in \Lambda_{p, k}(A)$. By Theorem $3.2, A_{j}$ has dimension at least $3 k-2$ ensuring that $\Lambda_{k}(A) \neq \emptyset$. The last assertion follows.

If $A \in H_{n}$ and $n \leq(p+1) k-1$, it follows from Theorem 3.5 that $\Lambda_{p, k}(A)$ consists of matrices in $H_{p}$ with eigenvalues $a_{k}, a_{2 k}, \ldots, a_{p k}$. Therefore, $\Lambda_{p, k}(A)$ is convex if and only if $a_{k}=a_{p k}$. If $n \geq(p+1) k$, we have the following.

Theorem 3.8 Suppose $n \geq(p+1) k$ and $A \in H_{n}$. Then $\Lambda_{p, k}(A)$ is convex if and only if

$$
\lambda_{k}(A)=\lambda_{p k}(A) \quad \text { and } \quad \lambda_{n-p k+1}(A)=\lambda_{n-k+1}(A) .
$$

Proof. Suppose $\lambda_{k}(A)=\lambda_{p k}(A)$ and $\lambda_{n-p k+1}(A)=\lambda_{n-k+1}(A)$. Then by Theorem 3.5,

$$
\Lambda_{p, k}(A)=\left\{B \in H_{p}: \lambda_{n-k+1}(A) \leq \lambda_{j}(B) \leq \lambda_{k}(A) \text { for all } 1 \leq j \leq p\right\},
$$

which is clearly convex.

We will prove the converse by contradiction. Suppose first that $\lambda_{p k}(A)<\lambda_{k}(A)$. Let $B=$ $\operatorname{diag}\left(\lambda_{k}(A), \lambda_{2 k}(A), \ldots, \lambda_{p k}(A)\right)$ and $R=\left[r_{i j}\right]_{i, j=1}^{p}$ where $r_{i j}=1$ if $i \equiv j+1 \bmod (p)$, and $r_{i j}=0$ otherwise. For $1 \leq j \leq p$, let $B_{j}=R^{j-1} B\left(R^{t}\right)^{j-1}$. Then $B_{j} \in \Lambda_{p, k}(A)$ for all $1 \leq j \leq p$ but $\left(\sum_{j=1}^{p} B_{j}\right) / p=\operatorname{diag}(b, \ldots, b)$, where $b=\left(\lambda_{k}(A)+\lambda_{2 k}(A)+\cdots+\lambda_{p k}(A)\right) / p>\lambda_{p k}(A)$. Therefore, $\operatorname{diag}(b, \ldots, b) \notin \Lambda_{p, k}(A)$ and $\Lambda_{p, k}(A)$ is not convex. The proof for the case when $\lambda_{n-p k+1}(A)>$ $\lambda_{n-k+1}(A)$ is similar.

We have obtained only some basic results for the $(p, k)$ numerical range. There are many problems that deserve further study. We mention some of them in the next section.

\section{Further research}

In [7], Fan and Pall study the conditions on which a $k \times k$ normal matrix $B$ can be imbedded into an $n \times n$ normal matrix with prescribed eigenvalues. They give a necessary and sufficient condition for the case when $k=n-1$. Since then, the problem has attracted the attention of many researchers, 
e.g. see $[2,10,17,21,23]$ but, the question for $1<k<n-1$ remains open. More specifically, it would be interesting to solve the following.

Problem 4.1 Determine all possible $k \times k$ principal submatrices of $U^{*} A U$ for a given $n \times n$ matrix A. Equivalently, we want to find all compressions of $A$.

When $A$ is Hermitian, Theorem 1.1 provides the answer. We can also study the above problem for general $A \in M_{n}$. Suppose $B \in M_{k}$ is a principal submatrix of $A$. Let $A=A_{1}+i A_{2}$ and $B=B_{1}+i B_{2}$ be the Hermitian decomposition of $A$ and $B$. Then $\cos t B_{1}+\sin t B_{2}$ is a submatrix of $\cos t A_{1}+\sin t A_{2}$ for all $t \in \mathbb{R}$ so that their eigenvalues satisfy the interlacing inequalities (1). Thus, we have an infinite family of inequalities. But the converse is not true as shown by the following example.

Example 4.2 Let $A=\left[\begin{array}{cccc}1 & 0 & 0 & 0 \\ 0 & 1 & 0 & 0 \\ 0 & 0 & i & 0 \\ 0 & 0 & 0 & -i\end{array}\right]$ and $B=\left[\begin{array}{cc}0 & \sqrt{2} \\ 0 & 0\end{array}\right]$. Then direct computation shows that for every $t \in \mathbb{R}$, the eigenvalues of $\cos t B_{1}+\sin t B_{2}$ and $\cos t A_{1}+\sin t A_{2}$ satisfy the interlacing inequalities (1). Since $\|B\|=\sqrt{2}>1=\|A\|, B$ cannot be a principal submatrix of $U^{*} A U$ for any unitary $U$.

A principal submatrix of a normal matrix may not be normal. Nevertheless, it would be interesting to determine all $k \times k$ normal principal submatrices of $U^{*} A U$ for a given normal matrix $A \in M_{n}$.

The results in Section 3 are motivated by problems in quantum information science. In connection to Theorem 3.6, it would be interesting to answer the following.

Problem 4.3 Determine the optimal $n$ so that $\Lambda_{p, k}(A)$ is non-empty for any $A \in M_{n}$.

It is known that $\Lambda_{k}(A)$ is always convex, and Theorem 3.8 gives the condition for $\Lambda_{p, k}(A)$ to be convex for Hermitian $A$. It is natural to ask the following.

Problem 4.4 Determine $A \in M_{n}$ such that $\Lambda_{p, k}(A)$ is convex.

Note that even for the special case when $A$ is normal, the problems 4.3 and 4.4 are still open and highly nontrivial.

\section{References}

[1] R. Bhatia, Perturbation bounds for matrix eigenvalues. Pitman Research Notes in Mathematics 162, Longman Scientific and Technical, New York, 1987.

[2] D. Carlson and E. Marques de Sa, Generalized minimax and interlacing theorems, Linear Multilinear Algebra 15 (1984), 77-103.

[3] M.D. Choi, Completely positive linear maps on complex matrices, Linear Algebra Appl. 10 (1975), 285-290. 
[4] M.D. Choi, M. Giesinger, J.A. Holbrook, and D.W. Kribs, Geometry of higher-rank numerical ranges, Linear and Multilinear Algebra 56 (2008), 53-64.

[5] M.D. Choi, N. Johnston, and D.W. Kribs, The multiplicative domain in quantum error correction, Journal of Physics A: Mathematical \& Theoretical 42 (2009), 245303.

[6] M.D. Choi, D.W. Kribs, and K. Życzkowski, Quantum error correcting codes from the compression formalism, Rep. Math. Phys. 58 (2006), 77-91.

[7] K. Fan and G. Pall, Imbedding conditions for Hermitian and normal matrices, Canad. J. Math. 9 (1957), 298-304.

[8] W. Fulton, Eigenvalues, invariant factors, highest weights, and Schubert calculus, Bull. Amer. Math. Soc. 37 (2000), 209-249

[9] R.A. Horn and C.R. Johnson, Topics in matrix analysis, Cambridge University Press, Cambridge, 1991.

[10] K.D. Ikramov and L. Elsner, On normal matrices with normal principal submatrices, J. Math. Sci. 89 (1998), 1631-1651.

[11] D.W. Kribs, R. Laflamme, D. Poulin, and M. Lesosky, Operator quantum error correction, Quantum Information \& Computation 6 (2006), 382-399.

[12] C.K. Li and R.C. Li, A note on eigenvalues of perturbed Hermitian matrices, Linear Algebra Appl. 395 (2005), 183-190.

[13] C.K. Li and R. Mathias, The Lidskii-Mirsky-Wielandt Theorem: additive and multiplicative versions, Numerische Mathematik 81 (1999), no. 3, 377-413.

[14] C.K. Li, Y.T. Poon, and N.S. Sze, Condition for the higher rank numerical range to be non-empty, Linear and Multilinear Algebra 57 (2009), 365-368.

[15] C.K. Li and N.S. Sze, Canonical forms, higher rank numerical ranges, totally isotropic subspaces, and matrix equations, Proc. Amer. Math. Soc. 136 (2008), 3013-3023.

[16] C.K. Li and N.K. Tsing, On the $k^{\text {th }}$ matrix numerical range, Linear and Multilinear Algebra 28 (1991), 229-239.

[17] S.M. Malamud, Inverse spectral problem for normal matrices and the Gauss-Lucas theorem, Trans. AMS 357 (2005), 40-43.

[18] A.W. Marshall and I. Olkin, Inequalities: Theory of majorization and its applications, Academic Press, Inc., New York-London, 1979.

[19] L. Mirsky, Matrices with prescribed characteristic roots and diagonal elements, J. London Math. Soc. 33 (1958) 14-21. 
[20] M.A. Nielsen and I.L. Chuang, Quantum Computation and Quantum Information, Cambridge, 2000.

[21] S.V. Savchenko, Normal matrices and their principal submatrices of co-order one, Linear Algebra Appl. 419 (2006), 556-568.

[22] G. Stewart and J.G. Sun, Matrix perturbation theory, Academic Press, Boston, 1990.

[23] R.C. Thompson, Principal submatrices of normal and Hermitian matrices, Illinois J. Math. 10 (1966), 296-308. 